Omega-P, Inc.

Final Report on STTR Phase I grant DE-FG02-08ER 86354

Topic 49b: Advanced concepts and technology for high energy accelerators:

NOVEL DEVICE AND INSTRUMENTATION DEVELOPMENT

Omega-P, Inc.

291 Whitney Avenue - Suite 401

New Haven, CT 06511

\title{
HIGH-CURRENT COLD CATHODE FIELD EMISSION ARRAY FOR ELECTRON LENS APPLICATION
}

\author{
Principal Investigator: J. L. Hirshfield \\ tel:(203)789-1165, e-mail: jay@ omega-p.com \\ FINAL REPORT \\ Phase I STTR grant DE-FG02-08ER 86354 \\ Topic 49b: Advanced Concepts And Technology For High Energy Accelerators
}

\begin{abstract}
During Phase I, the following goals were achieved: (1) design and fabrication of a novel, nano-dimensional CNT field emitter assembly for high current density application, with high durability; (2) fabrication of a ceramic based micro channel plate (MCP) and characterization of its secondary electron emission; and (3) characterizing the CNT/MCP cathode for high field emission and durability. As a result of these achievements, a relatively high current density of $\sim 1.2 \mathrm{~A} / \mathrm{cm}^{2}$ from a CNT cathode and single channel MCP were measured. The emission current was also extremely stable with a peak-to-peak variation of only $1.8 \%$. The emission current could be further enhanced to meet requirements for electron lens applications by increasing the number of MCP channels. A calculation for maximum possible current density with a 1200 channel $/ \mathrm{cm}^{2} \mathrm{MCP}$, placed over a cathode with 1200 uniformly functioning CNTs, would be $\sim 1.46 \mathrm{kA} / \mathrm{cm}^{2}$, neglecting space charge limitations. Clearly this level of emission is far greater than what is needed for the electron lens application, but it does offer a highly comforting margin to account for sub-standard emitters and/or to allow the lesser challenge of building a cathode with fewer channels $/ \mathrm{cm}^{2}$. A satisfactory goal for the electron lens application would be a controllable emission of 2-4 mA per channel in an ensemble of 8001200 uniformly-functioning channels $/ \mathrm{cm}^{2}$, and a cathode with overall area of about $1 \mathrm{~cm}^{2}$.
\end{abstract}

\section{CONTENTS}

$\begin{array}{llr} & \text { INTRODUCTION } & 2 \\ \text { II. } & \text { TECHNICAL APPROACH } & 4 \\ \text { III. } & \text { CONCLUSIONS AND ACKNOWLEDGEMENTS } & 14 \\ \text { IV. } & \text { REFERENCES } & 15\end{array}$

Work on this STTR project was carried out at Florida International University by Raghunandan Seelaboyina, Indranil Lahiri, and Professor Wonbong Choi; and at Omega-P, Inc. by Dr. J. L. Hirshfield, Principal Investigator. The proposal was written by Professor Choi and Dr. Hirshfield.

December 29, 2012 


\section{Omega-P, Inc.}

Final Report on STTR Phase I grant DE-FG02-08ER 86354

Topic 49b: Advanced concepts and technology for high energy accelerators:

NOVEL DEVICE AND INSTRUMENTATION DEVELOPMENT

\section{INTRODUCTION}

The DoE 2008 SBIR/STTR Phase I Program Solicitation requested grant applications under Topic 49b Advanced concepts and technology for high energy accelerators: Novel Device and Instrumentation Development, where "...applications are sought for the development of electromagnetic ... charged particle optical elements for particle beam focusing." Omega-P, Inc. submitted a proposal in response, and was granted STTR grant DE-FG02-08ER 86354, entitled "High current cold cathode field emission array for electron lens applications." The overall goals of the R\&D program included the development, design and demonstration of a new type of triggered field emission carbon nanotube cold cathode suitable for application to generate the beam for an electron lens for a high-energy proton collider. Preliminary design work and testing were carried out during Phase I, while fabrication and testing of the cathode, and measurements of parameters of the electron beam it would generate, were to be carried out during Phase II. However, the Phase II grant request was not approved, so this report summarizes all work done on the topic by Omega-P, Inc. and its collaborator Florida International University.

DoE support for this work was structured as an STTR grant, since significant collaboration between Omega-P, Inc. and Florida International University (FIU) was critical to the research. In particular, Omega-P determined beam control parameters, current density and geometrical requirements for the cathode to be consistent with application in an electron lens, and conducted laboratory tests of the cold cathode; while FIU designed and developed the triggered field emission cold cathode itself. This collaboration built upon Omega-P's expertise in sophisticated electron guns for high-power microwave aspects of accelerator physics, including development of guns with extremely high transverse beam area compression for RF sources for accelerator application; coupled with FIU's expertise on high-current-density carbon nanotube field emitters. Omega-P and FIU coordinated respective tasks and worked jointly to analyze the results.

The luminosity of circular proton-antiproton colliders is limited by the effects of electromagnetic interaction of one beam on the particles of the other beam, which leads to a blowup of beam sizes, a loss of beam intensities, and unacceptable background rates in the high energy physics detectors. Operation with a greater number of bunches allows a proportional increase of luminosity but requires careful spatial separation of two beams everywhere except at the main intersection points. Long-range (as opposed to head-on) interactions of separated beams are also nonlinear and contribute to the limit on collider performance. These long-range effects usually vary from bunch to bunch, making their treatment even more cumbersome. Electron lenses were proposed for compensation of both long-range and head-on beam-beam effects in the Fermilab Tevatron collider, as described by Shiltsev et al [1-3]. The lens employed a low energy beam of electrons which collided with the high-energy bunches over an extended length. The main advantages of the electron lens compensation are: (a) the electron beam acts on high- 
energy beams only through electromagnetic forces (no nuclear interaction), eliminating radiation issues; $(b)$ fresh electrons interact with the high-energy particles on each turn, leaving no possibility for coherent instabilities; $(c)$ the electron current profile (and thus the electromagnetic field profile) can easily be changed for different applications; and $(d)$ the electron-beam current can in principle be adjusted between each of the bunches, equalizing the bunch-to-bunch differences and optimizing the performance of all of the bunches in multi-bunch colliders.

Electron lenses were successfully tested at the Fermi National Accelerator Laboratory (FNAL) Tevatron collider [3], demonstrating the strong potential of this method of beam-beam interaction compensation. The electron guns currently used in Tevatron electron lenses employ both 10- and 15-mm diameter convex dispenser cathodes. The lenses operate with 500-700 ns pulses and peak electron currents of 0.6-1A. The pulse width, the amplitude and the repetition rate $(\sim 50 \mathrm{kHz})$ is limited by the capacity of high voltage pulse generators that are used. In the present configuration the biggest problem is the necessity to switch a voltage of several kilovolts at high repletion rate, so as to provide pulses compatible with the proton (antiproton) bunch length. If there would be a way to modulate the electron current with much lower voltage it would give the possibility to go to much higher repetition rates and shorter pulses, which in turn would give the possibility to correct the beam-beam effects in all the bunches separately. The transverse charge density distribution of the electron beam is crucial for this application. The electron gun should be capable of generating Gaussian-like profiles and a profile with a flat top and smooth radial edges (SEFT). It is not inconceivable that a proven electron lens as described here could also find application with LHC [3]. The table below summarizes typical electron beam parameters.

Table I. Parameters of beam for electron lens.

\begin{tabular}{|c|c|}
\hline cathode voltage & $10 \mathrm{kV}$ \\
\hline peak electron current & $2-3 \mathrm{~A}$ \\
\hline current density distribution & SEFT/Gaussian \\
\hline maximum DC dark current & $0.01 \mathrm{~A}$ \\
\hline pulse length & $100 \mathrm{~ns}$ flat top \\
\hline maximum repetition frequency & $2.5 \mathrm{MHz}$ \\
\hline
\end{tabular}

New understanding and implementation of advanced laboratory techniques have recently allowed fabrication of triggered carbon nanotube (CNT) field emitters that show great promise as cathodes for a variety of accelerator applications. Field emission sources have not proven practical in earlier attempts to incorporate them into cathode units for electron guns, due to their fragility and susceptibility to destruction from ion bombardment and/or unavoidable vacuum arcs during gun conditioning. But the novel approach described here for utilizing cold fast-triggered cathode field emission CNT's, coupled with secondary current channel multiplication in an outer barrier that can protect the $\mathrm{CNT}^{\prime} \mathrm{s}$, offer new opportunities for building improved, high rugged 


\section{Omega-P, Inc.}

Final Report on STTR Phase I grant DE-FG02-08ER 86354

Topic 49b: Advanced concepts and technology for high energy accelerators:

NOVEL DEVICE AND INSTRUMENTATION DEVELOPMENT

cathodes for many vacuum electronics applications, including improved guns for electron lenses for circular colliders such as the Tevetron and LHC.

This report summarizes results obtained during the Phase I STTR grant period by Omega-P, Inc. and Florida International University towards development of a high-current cold cathode field emission array that could meet the above summarized requirements for electron lens applications.

\section{TECHNICAL APPROACH}

The objective of the research described here is to address requirements for an electron lens by developing a cold cathode field emission system employing an ensemble of aligned carbon nanotube (CNT) emitters in conjunction with a ceramic electron multiplier micro-channel plate (MCP). The field emitters are to be subject to fast control through use of low-voltage gates, so as to allow rapid adjustment of beam current and/or of current density profile.

CNT emitters, even prior to work described here, have operated with relatively high current density $[4,5,6]$. But this large current density was obtained by randomly distributing CNT emitters within a small area, with only a small fraction of the total available nanotubes participating in the electron emission. However, the challenge for applications such as the electron lens is to achieve a reliable, rapidly-controllable current density in the range of 10 $\mathrm{A} / \mathrm{cm}^{2}$, using a robust long-lifetime cathode structure. Moreover, to increase the total emission current, a large number of emitters must operate simultaneously with maximum field enhancement. To meet these requirements, new emitters with low work function, high field enhancement, and very good shape uniformity are needed. It is also critical that novel device design for a high current density together with good protection against vacuum arc damage and high thermal dissipation be developed to maximize the emitter performance and reliability. Furthermore, rapid low-level control of individual CNT emitters is essential, both to control the overall current, and to tailor the current distribution across the beam.

So far, the highest total current of electron emission from a CNT field emission cathode has been 0.1- $0.8 \mathrm{~A}$ [7]. Carbon nanotube emitters have shown a reasonably impressive current density, as high as $4 \mathrm{~A} / \mathrm{cm}^{2}$ [4]. A realistic practical challenge is to demonstrate a total current in the range of $10 \mathrm{~A}$ from an emitting area of $1 \mathrm{~cm}^{2}$ or less, with the added possibility of rapid lowvoltage control of total current and of current density profile. To meet those requirements, powerful gated emitters which are uniform and capable of high current emission and yet are robust enough to endure intense field emission environment are needed. A schematic diagram of a version of the cathode under development is shown in Fig. 1.

Strong fields that are present in conventional field emitters will accelerate ions from residual gas ionization towards the emitters, leading to erosion and finite cathode lifetime, while unavoidable vacuum arcs can lead to irreversible cathode damage. The unique cold cathode 


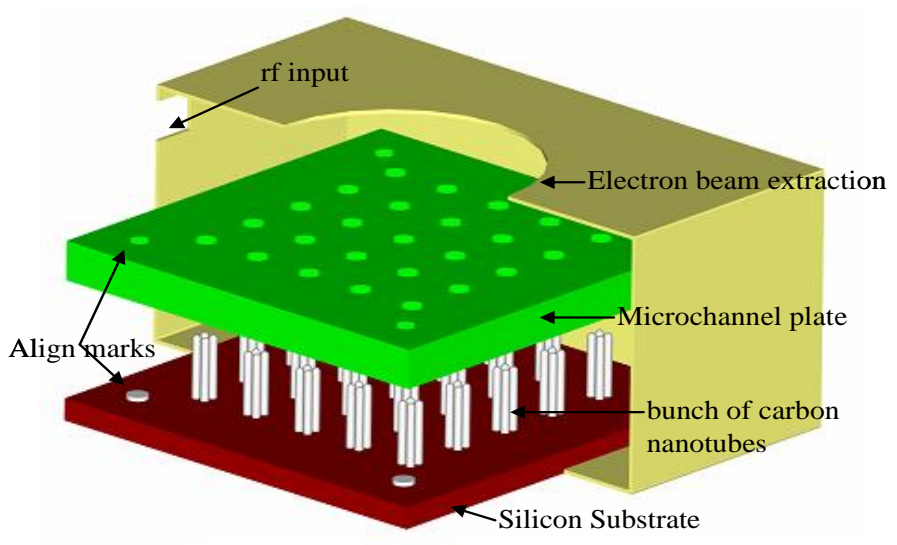

Fig. 1. Schematic of the proposed novel cold cathode for high current density, using carbon nanotube emitters and electron multiplier. The port labeled "rf input" is to symbolize an input for external current control, whether by external RF fields or via FET's on the nanotubes.

design being developed in this project places electron multipliers on top of the emitters to increase electron current density and to protect the cathode from plasma puffs and arcing. The solid ceramic structure of the electron multiplier will act as a rugged shield, providing significant protection of the nanotubes against hostile events. The inability of field emitter arrays (FEAs) to emit uniformly or to achieve a linear scaling of emission current with array size is due to the exponential relationship between current and voltage (I-V). The exponential part of the FowlerNordheim equation contains both the field enhancement term and the work function term. Small changes in field enhancement, i.e. tip sharpness or emitter height, and changes in the local work function due to adsorbates cause an exponential changes in emission current. Even in the bestmade arrays, the sharpest, cleanest, and tallest tips emit first, and most of the current is produced from a very small percentage of tips. However, as the anode potential is increased these overburdened tips will fail catastrophically before the rest of the tips can contribute any significant current. A major limitation to the application of field emitters is the lack of emission stability. Fluctuations in emission current by a factor of more than two are common for most emitters not operating under ultra-clean, ultra high vacuum conditions. This is not only due to the constituents of the given vacuum, but also the instability of the emitter materials themselves while subjected to high fields and currents. Novel electron multiplier and high surface resistance emitters together with novel materials and device design are needed for the next generation vacuum devices, including guns for electron lenses.

The approach followed in the current project addresses and overcomes both the current density limits and vacuum breakdown problems in parallel, and moreover provides the opportunity for building into the cathode structure rapid control of current control using lowvoltage gates at each emitter. Critical issues involved include the emitter material and cathode design to obtain high currents, survival of cathodes in the presence of arc damage, ion 
bombardment, and operation in a space-charge limited mode. This research should lead to major breakthrough in the creation of novel cold cathodes, electron multiplier and practical remediation of vacuum breakdown problems in high power vacuum devices, while developing much better understanding of the emission behavior, new emitter materials, and modeling techniques. The powerful field emitters being designed and fabricated in this project for electron lens applications can also be useful for a variety of important applications such as flat panel field emission displays, $\mathrm{x}$-ray, high power microwave and solid state lighting in general. These powerful emitters offer a major advantage of being able to operate at very moderate conditions, which enables more consistent and reliable performance at less power and longer life, and requires less critical demands in electronic circuit design and operational criteria.

\section{IIa. Specific technical objectives}

The goals of this STTR project are to design, build, and test for electron lens applications a cold cathode embodying an array of gated field emitting carbon nanotubes. Successful tests of this novel cathode by Omega-P and Florida International University (FIU) is intended to lead, during Phase II, to installation of a prototype gun on a test stand at FNAL for tests by FNAL personnel to evaluate the utility of the gun and cathode for an electron lens configuration. Specific objectives include rapid control of cathode current and cathode current density profile, with a response time of the order of $100 \mathrm{~ns}$. Reliability, robustness and pulse-to-pulse reproducibility are considered essential for the electron lens application, so design considerations will be with these objectives in mind. As an STTR collaboration, the research partners Omega-P and FIU share responsibility for meeting these goals: Omega-P sets specifications for the prototype cathode to be suitable for electron lens applications, including pulsed power supply requirements, and will design and build the test stand for evaluating cathode properties prior to undertaking tests at FNAL; FIU is applies its expertise in development of the carbon nanotube array and channel current multipliers with an emitter density appropriate for a $2-3 \mathrm{~A}, 10 \mathrm{kV}$ beam; and will develop gate controls for the nanotubes so as to tailor the current distribution and the current magnitude itself with $100 \mathrm{~ns}$ response time; and will develop the cathode assembly

including a hard ceramic outer face to protect the CNTs and current amplifier channels from ion back bombardment.

During Phase I, the following goals were achieved: (1) design and fabrication of a novel, nano-dimensional CNT field emitter assembly for high current density application, with high durability; (2) fabrication of a ceramic based micro channel plate (MCP) and characterization of its secondary electron emission; and (3) characterizing the CNT/MCP cathode for high field emission and durability. As a result, a relatively high current density of $\sim 1.2 \mathrm{~A} / \mathrm{cm}^{2}$ from a CNT cathode and single channel MCP were measured. The emission current was also extremely stable with a peak-to-peak variation of only $1.8 \%$. The emission current can be further enhanced to meet the cathode requirements for electron lens applications, by increasing the number of MCP 
channels. A simple calculation for maximum possible current density with a 1200 channel/ $/ \mathrm{cm}^{2}$ MCP, placed over a cathode with 1200 uniformly functioning CNTs, would be $\sim 1.46 \mathrm{kA} / \mathrm{cm}^{2}$, neglecting space charge limitations. Clearly this level of emission is far greater than what is needed for the electron lens application, but it does offer a highly comforting margin to account for sub-standard emitters and/or to allow the lesser challenge of building a cathode with fewer channels $/ \mathrm{cm}^{2}$. A satisfactory goal for the electron lens application would be a controllable emission of 2-4 mA per channel in an ensemble of 800-1200 uniformly-functioning channels $/ \mathrm{cm}^{2}$, and a cathode with overall area of about $1 \mathrm{~cm}^{2}$.

Novel electron multiplier and high surface resistance emitters, together with novel materials and device design, will be provided for cathode elements for the electron lens application. In addition to improving the total field emission current and its stability, the cathode emission profile will also be controlled by adding a gate electrode (i.e., a series FET) in between the MCP and cathode, or at the base of the CNT. The gate electrode will be designed to control the CNT emitters individually or as a group, to quickly modify the beam's transverse current density profile, for further adjustment of focusing properties. Since the gate to cathode distance will be smaller than the MCP to cathode distance, these arrangements will allow relatively small gate voltages to exert significant control over the field emission. This novel structure has great potential to be used in other applications, such as in high power microwave devices, due to its high, stable emission current and fast current control.

\section{IIb. Microchannel plate fabrication}

The fabrication procedure of the ceramic template for the MCP is demonstrated in Fig. 2. The process involves the following steps:

(a) $\sim 620 \mu \mathrm{m}$ thick alumina $\left(\mathrm{Al}_{2} \mathrm{O}_{3}\right)$ un-sintered tape was cut into required size in which, $\sim 300 \mu \mathrm{m}$ diameter channels were formed by laser drilling.

(b) The laser processed green sheets were then sintered at $1550^{\circ} \mathrm{C}$, resulting in $\sim 500 \mu \mathrm{m}$ thick MCP template with channels of $\sim 275( \pm 15) \mu \mathrm{m}$ diameter (reduced dimensions of the microchannel template are due to shrinkage of green bodies during sintering).

(c) The sintered templates with microchannels were, later, treated with $\mathrm{H}_{3} \mathrm{PO}_{4}$ to clean the channels from any residual slag or debris, formed during laser processing. In addition, the acid treatment increased the roughness of the $\mathrm{Al}_{2} \mathrm{O}_{3}$ surface, resulting in improved adhesion of the deposited $\mathrm{Cu}, \mathrm{Fe}$ layer.

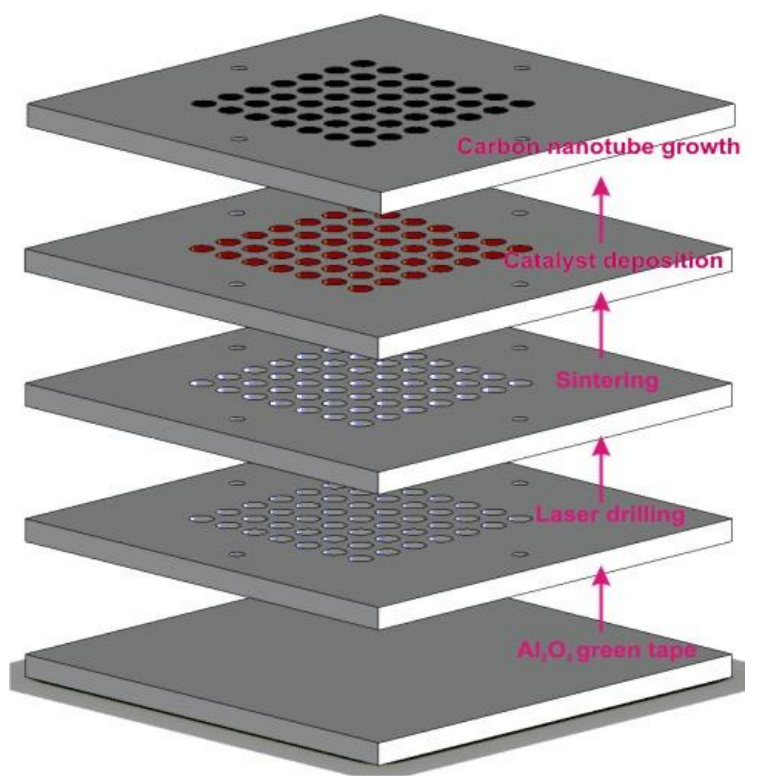

Fig. 2. MCP fabrication procedure [8]. 
(d) Finally $\mathrm{MgO}$ was deposited on the $\mathrm{Cu}$ or CNT layer by electrophoretic deposition (EPD) technique. For EPD deposition, an alcohol solution with nano-MgO powder was utilized.

The SEM image of MCP channel cross-section, with uniformly grown CNTs (instead of depositing $\mathrm{Cu}$ layer, $\mathrm{CNTs}$ were grown) along the $\mathrm{MgO}$ coated channel, is shown in Fig. $3 \mathrm{a}$ and the inset shows the magnified SEM image of MgO-coated CNTs. The TEM image of the nanotubes, coated with $\mathrm{MgO}$, is shown in Fig. 3b. Samples for TEM analysis were prepared by scraping off the nanotubes from the channel and the surface. It can be observed from the image that the nanotube is coated with more $\mathrm{MgO}$ on one end, which might have been the top surface of the nanotubes, grown along the channel. This is expected for nanotubes grown horizontally on a surface. In this case, the end, depleted in $\mathrm{MgO}$ coating, might have been the end attached to the channel i.e. $\mathrm{Al}_{2} \mathrm{O}_{3}$ surface. The EDX spectrum of the $\mathrm{MgO}$-coated CNT (Fig. 3b) shows presence of $\mathrm{Mg}$ and $\mathrm{O}$. The length and diameter of the nanotubes on the MCP were $\sim 40-50 \mu \mathrm{m}$ and $\sim 20-30 \mathrm{~nm}$, respectively and the $\mathrm{MgO}$ coating thickness varied from $\sim 20-100 \mathrm{~nm}$. SEM and TEM investigations, made on several samples, agreed with images shown in Fig. 3.
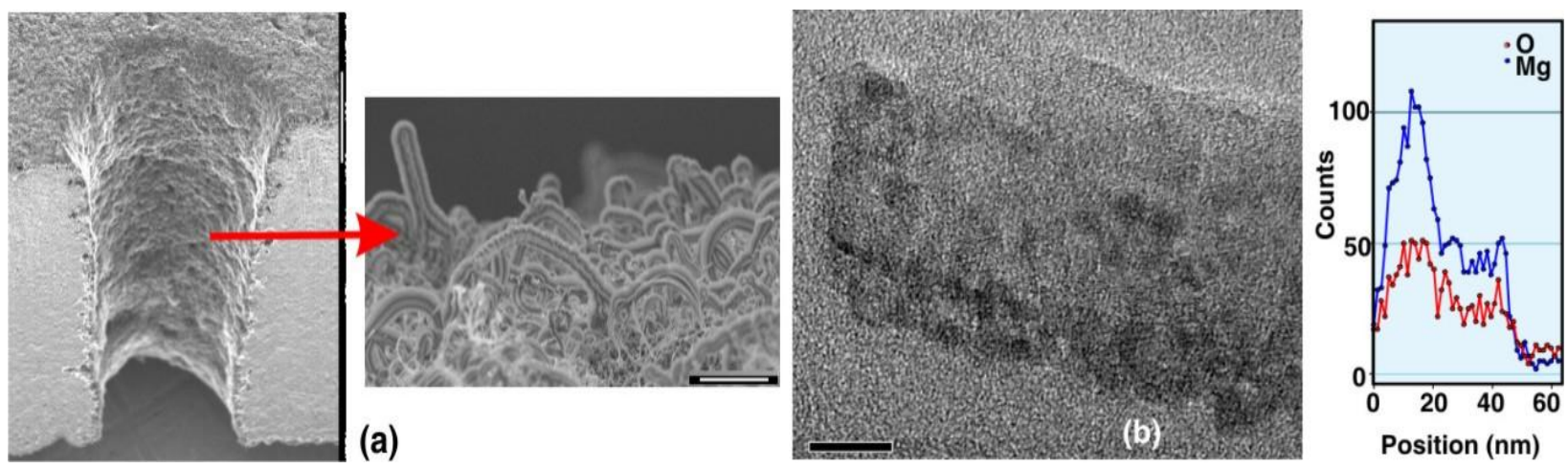

Fig. 3. SEM (scale:100 $\mu \mathrm{m}$ ) image of (a) cross-section of MCP channel showing uniform layer of MgO-coated CNTs (inset) magnified view of MgO-coated CNT along the channel (b)

HRTEM image (scale: $10 \mathrm{~nm}$ ) of $\mathrm{MgO}$-coated CNT, higher amount of $\mathrm{MgO}$ coating can be seen on side of the CNT from the image; which might be the nanotube surface facing away from the channel surface (right) corresponding EDX spectrum i.e. line scan along the MgOcoated CNT showing the presence of $\mathrm{MgO}$, higher amount of $\mathrm{MgO}$ coating on one side of nanotube can also be observed from the scan [9].

\section{IIc. Synthesis of carbon nanotube array}

Vertically aligned CNT arrays on porous-silicon (Si), shown in Fig. 4a, were utilized to determine the FE current enhancement from the MCP. Porous-Si substrates for CNT growth were prepared by anodization of 2 inch diameter and $300 \mu \mathrm{m}$ thick p-type $\mathrm{Si}<100\rangle$, with a resistivity of $0.008-0.02 \mathrm{ohm}-\mathrm{cm}$. Anodization was performed in an electrolyte, under galvanostatic i.e. current-controlled, conditions. The electrolyte consisted of a 1:1 mixture (by 
volume) of $48 \mathrm{wt} \% \mathrm{HF}$ and 100\% ethanol. An aluminum foil pressed on the backside of the sample served as an ohmic contact and a platinum wire served as counter electrode. Anodization was carried out at a rate of $10 \mathrm{~mA} / \mathrm{cm}^{2}$ for $\sim 5$ minutes, resulting in a nano porous-Si layer, with a pore diameter of $\sim 15-20 \mathrm{~nm}$. Iron $(\mathrm{Fe})$ catalyst thin film $(\sim 10 \mathrm{~nm})$ was sputtered on the resulting porous-Si substrates through a shadow mask, followed by annealing for $\sim 12$ hours at $300^{\circ} \mathrm{C}$. Annealing under these conditions was observed to improve the contact of Fe catalyst with the nanopores. In addition, annealing was also observed to relieve the residual stress in the porous film, thus avoiding cracking during the nanotube growth. Annealed substrates were then placed in a thermal CVD system for the nanotube synthesis, followed by heating to $700^{\circ} \mathrm{C}$ in $\operatorname{Ar}(\sim 1000$ sccm) atmosphere. After the temperature was stabilized at $700^{\circ} \mathrm{C}$, Ar was replaced by $\mathrm{C}_{2} \mathrm{H}_{2}$ ( $\sim 1000 \mathrm{sccm}, 40$ minutes) precursor gas, followed by cooling to room temperature for $\sim 2$ hours.

Fig. 4(a) shows the SEM image of vertically grown MWNTs on porous-Si nanotemplate. The growth mode of nanotubes on the porous-silicon is base growth mode, similar to the one demonstrated by Fan et al [10]. To verify the growth mode, nanotubes were completely removed by reactive ion etching (RIE). Nanotube growth was observed on the resulting etched substrates without catalyst deposition, thus confirming the base growth (images not shown here). Vertical alignment of nanotubes, grown on porous-Si nanotemplates, is achieved by van der Walls force interaction between the adjacent nanotubes, forming highly rigid bundles from densely packed catalyst particles. The rigidity enables nanotubes to keep growing along the original direction i.e. normal to the substrate and even outermost nanotubes are held by inner nanotubes without branching away [10]. Length and diameter of the nanotubes were controlled by varying the precursor gas concentration and flow during the CVD growth. The average length and diameter of MWNT arrays, grown for 45 minutes, was $\sim 55 \mu \mathrm{m}$ and $\sim 15-20 \mathrm{~nm}$, respectively [11].

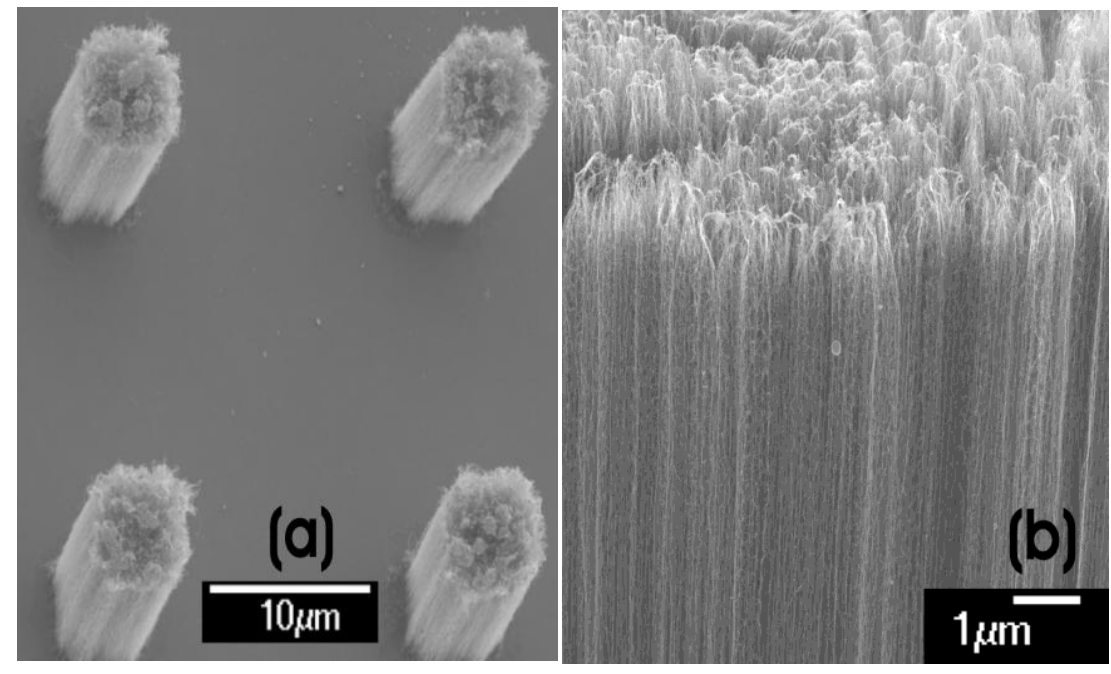

Figure 4. (a) SEM image of CNT pillars on porous silicon, (b) Magnified image of the pillar top surface 


\section{IId. Characterization of secondary electron emission}

The secondary electron yield (SEY) i.e. the ratio of the output to the input current of the novel MCP, with MgO-coated carbon nanotubes as secondary emissive layer, is demonstrated in Fig. 5. The experimental data for determining the SEY was acquired by retarding potential (RP) method. In RP method, MCP, with $\mathrm{MgO}$ coated CNT as secondary emissive layer, is kept at a negative potential with respect to the surrounding electrodes, simply to prevent the emitted SEs from returning back to the target. The current that passes through the MCP to ground is measured [12]. This current, $\mathrm{I}_{\mathrm{SP}}$, is the difference between the incident beam current, $\mathrm{I}_{\mathrm{P}}$, and the current of emitted secondary electrons (SEs), $\mathrm{I}_{\mathrm{SE}}$. The primary beam current, $\mathrm{I}_{\mathrm{P}}$, is measured with a Faraday cup mounted at the end of the e-gun (not shown here). The $\mathrm{I}_{\mathrm{p}}$ value is determined by focusing the beam to measure the maximum value of $I_{P}$. During the SEY measurement, the beam is unfocused, so that maximum possible area of MCP under investigation is exposed to the electron beam. The electron beam from the e-gun i.e. $I_{P}$, is kept constant throughout the measurement and negative potential of 10-1200 V (Fig. 5a) is applied, step-by-step, to the MCP, to measure the $\mathrm{I}_{\mathrm{SP}}$. Every time the potential of the $\mathrm{MCP}$ is varied, $\mathrm{I}_{\mathrm{P}}$ is also measured to check its stability. In our experiments, the $I_{P}$ value was extremely stable. The SEY is calculated from the relations $\left(1-\mathrm{I}_{\mathrm{SP}} / \mathrm{I}_{\mathrm{P}}\right)$ or $\left(\mathrm{I}_{\mathrm{SE}} / \mathrm{I}_{\mathrm{P}}\right)$. The total yield contains the elastically and in-elastically scattered electrons, as well as the low energy true secondaries. The represented data in Fig. 5a was measured from only a $4 \times 4$ array of $275( \pm 15) \mu \mathrm{m}$ diameter channels. The measurement area was limited due to the restriction of our experimental setup, i.e. the beam size and fixed sample stage facing the e-gun, Fig. 5b. As demonstrated in Fig. 5a, very high gain of $\sim 165$ was achieved with our novel MCP, when a primary beam current of $\sim 2.5 \mu \mathrm{A}$, from the e-gun, generated $412.5 \mu \mathrm{A}$ current as output. As seen in Fig. 5a, the secondary electron yield decreases after $1100 \mathrm{~V}$.

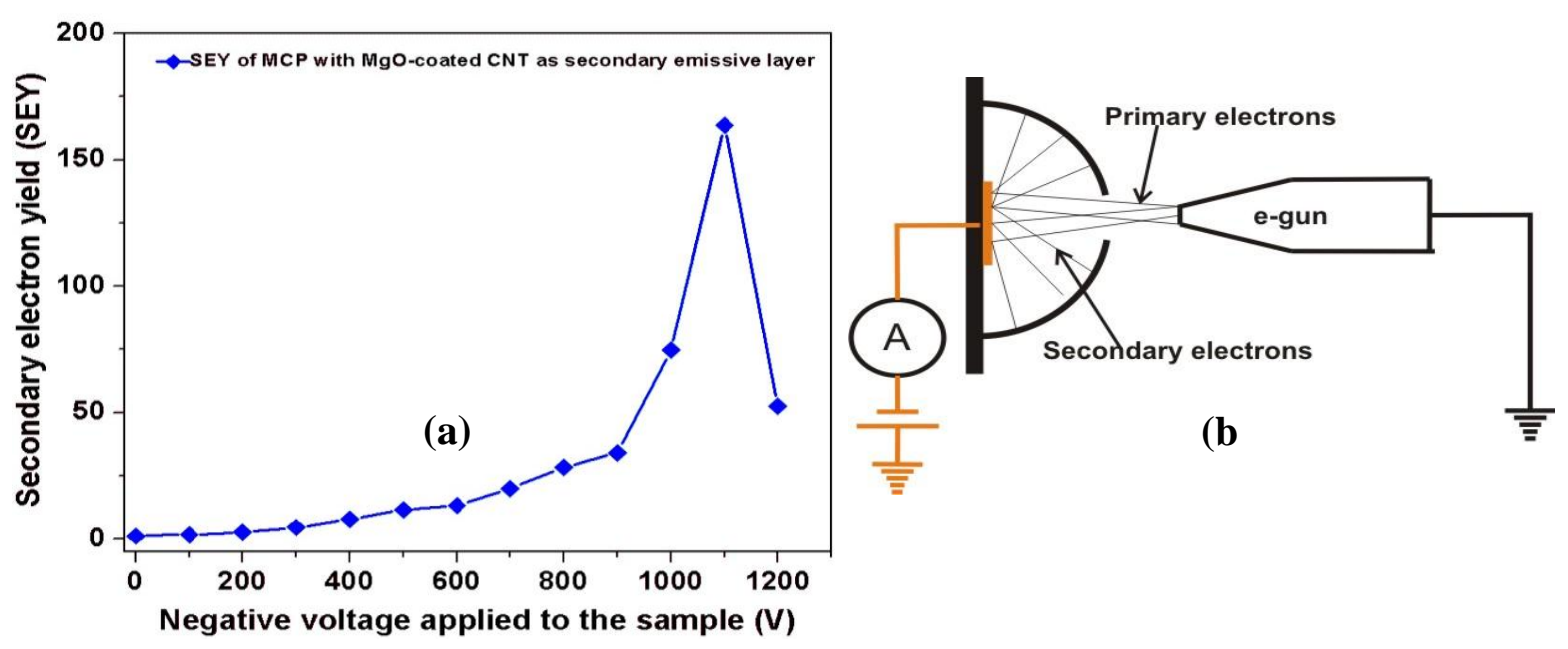

Fig. 5. (a) Secondary electron yield plot of a microchannel plate with $\mathrm{MgO}$-coated carbon nanotubes as secondary emissive layer (b) experimental set-up for acquiring secondary electron vield data by retarding potential method [9]. 
Final Report on STTR Phase I grant DE-FG02-08ER 86354

The penetration depth of primary electron is proportional to the magnitude of the applied potential. Initially, as the applied potential is increased, the secondary electron yield increases and once the penetration depth of primary electron exceeds the escape depth of the secondary electron, SEY decreases, as seen in Fig. 5a. The escape depth of secondary electrons is dependent on the material; for $\mathrm{MgO}$, it is $\sim 50-100 \mathrm{~nm}$ [12].

\section{IIe. Field emission from MCP/CNT emitters}

The MCP, with MgO-coated CNT as secondary emissive layer, was also tested for its gain, by placing it between a CNT cathode and an anode, as illustrated in Fig. 6a. When the electron multiplier structure i.e. the MCP, was placed between the cathode and anode, as shown in the figure 5a, a significant increase in current - 6.4 times, was achieved. The current (17.5 $\mathrm{mA}$ ) obtained with MCP, as shown in figure $5 \mathrm{~b}$, was achieved from an area of $0.4 \mathrm{~cm}^{2}$, which corresponds to a current density of $43.75 \mathrm{~mA} / \mathrm{cm}^{2}$. The high emission current was also very stable with a variation of $\sim 1.8 \%$ for 5 hours, as shown in figure $5 \mathrm{c}$. I-V measurements beyond 20 $\mathrm{mA}$ could not be performed due to the limitation of our voltage amplifier TREK 20/20C, which cannot measure peak current beyond $20 \mathrm{~mA}$. We believe that higher emission current can easily be achieved with our novel MCP/CNT cold cathode, using measurement electronics capable of measuring high current. This can be realized from the SEY characterization of the MCP, which showed a very high gain of 165 times. Inset of figure $5 \mathrm{~b}$ shows a bright and focused emission image with a MCP placed between the CNT cathode and anode. The well focused and bright spots in the emission image can be attributed to the electron multiplication and focusing of the MCP channels. The emission was very uniform, with almost 85-90\% of the channels emitting.

\section{IIf. Field emission from single channel MCP and cathode}

A novel carbon nanotube based cold cathode has recently been designed in our lab, which shows extremely high emission current. The emission current of the CNT cathode was further enhanced [13,14], when a MCP with single channel was placed over it (see Fig. 7a). The field emission I-V measurements were done in both DC (data not shown here) and AC modes; an ammeter (Keithley-2010) in the former and a current monitor (Pearson-4110) in the latter, was utilized to measure the emission current. FE in AC mode was measured with a sinusoidal wave, with frequency ranging from $400 \mathrm{~Hz}$ to $10 \mathrm{kHz}$ and the current signal was recorded on an oscilloscope (Agilent-MSO6034A). The signal generated from a function generator (Agilent3320A) was amplified by a voltage amplifier (Trek-20/20C), to generate the high voltage required for FE measurements in AC mode. The emission current of $4.3 \mathrm{~mA}$, as seen in Fig. 7a, with single channel MCP corresponds to a current density of $\sim 1.2 \mathrm{~A} / \mathrm{cm}^{2}$. Emission current was also very stable under AC bias for $\sim 2$ hours, as shown in Fig. $7 \mathrm{~b}$. 
Omega-P, Inc.

Final Report on STTR Phase I grant DE-FG02-08ER 86354

Topic 49b: Advanced concepts and technology for high energy accelerators:

\section{NOVEL DEVICE AND INSTRUMENTATION DEVELOPMENT}
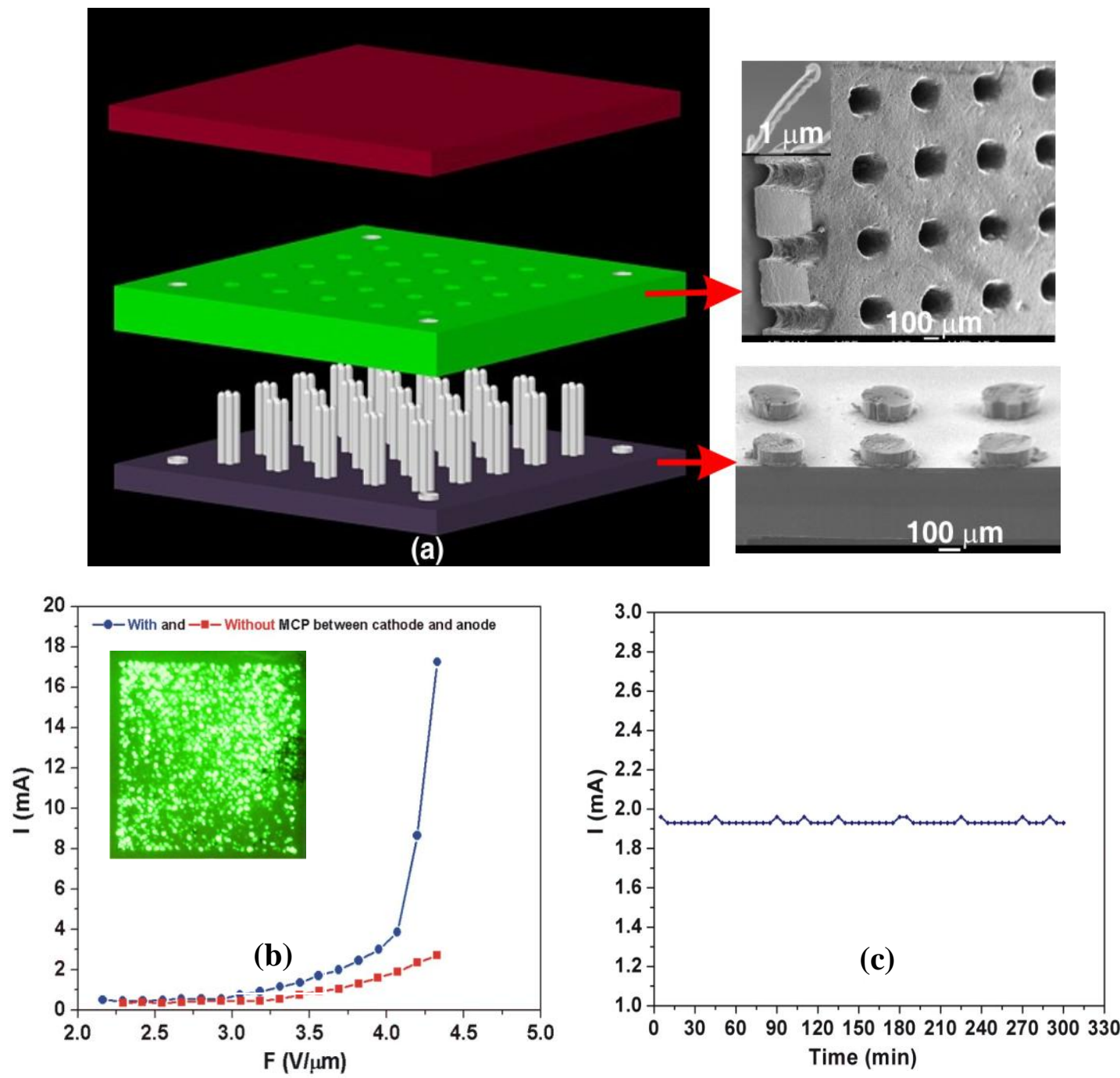

Fig. 6. (a) Schematic of the test structure with MCP placed between CNT cathode and anode, (right) the corresponding SEM images of MCP and CNT cathode (b) I-V data plot

with and without electron multiplier structure i.e. MCP between cathode and anode, emission current enhanced by $\sim 6.4$ times with MCP. The current density with MCP was $\sim 43.75 \mathrm{~mA} / \mathrm{cm}^{2}$;

(b) stable emission with a peak-to-peak variation of $\sim 1.8 \%$ for $\sim 5$ hours [9]. 
Omega-P, Inc.

Final Report on STTR Phase I grant DE-FG02-08ER 86354

Topic 49b: Advanced concepts and technology for high energy accelerators:

NOVEL DEVICE AND INSTRUMENTATION DEVELOPMENT
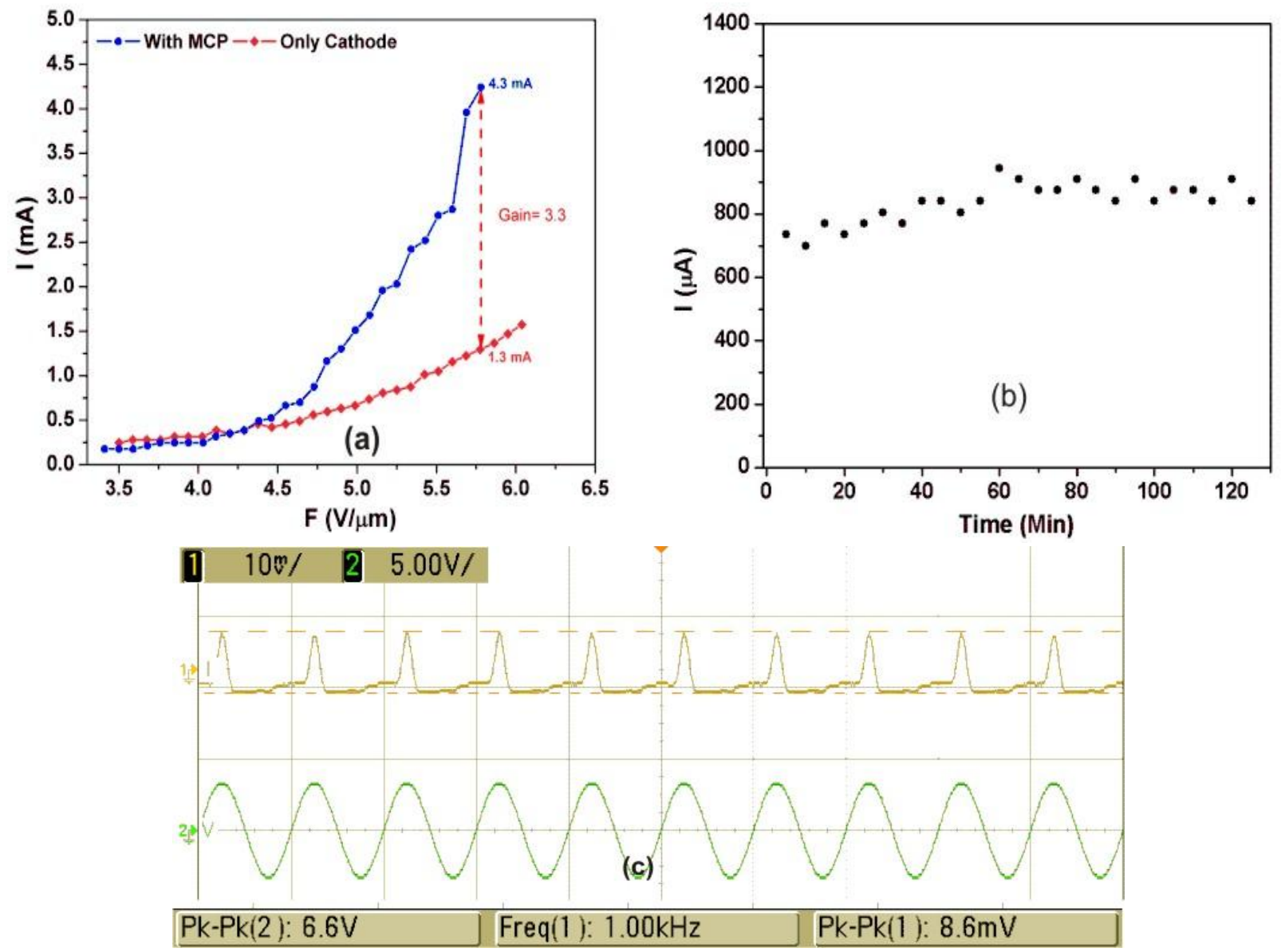

Fig. 7: (a) I-V response of the novel emitter structure with single channel MCP in AC bias (demonstrated values in the AC plot are RMS); (b) emission current stability of the single channel 3D cold cathode with MCP, under AC bias [1]; (c) oscilloscope pattern showing emission current (top signal) and applied voltage (bottom signal). Demonstrated signals are peak-to-peak, these values were converted to RMS to obtain a direct comparison with DC values. [1]. 
Omega-P, Inc.

Final Report on STTR Phase I grant DE-FG02-08ER 86354

Topic 49b: Advanced concepts and technology for high energy accelerators:

NOVEL DEVICE AND INSTRUMENTATION DEVELOPMENT

\section{CONCLUSIONS AND ACKNOWLEDGMENT}

During Phase I, the following goals were accomplished: design and fabrication was achieved of a novel, nano-dimensional CNT field emitter assembly for high current density application, with high durability; fabrication was achieved of a ceramic based micro channel plate (MCP) and characterization of its secondary electron emission; and

characterization was achieved for high field emission and durability of the CNT/MCP cathode.

As a result of these achievements, a relatively high current density of $\sim 1.2 \mathrm{~A} / \mathrm{cm}^{2}$ from a $\mathrm{CNT}$ cathode and single channel MCP were measured. The emission current was also extremely stable with a peak-to-peak variation of only 1.8\%. The emission current could be further enhanced to meet the cathode requirements for electron lens applications, by increasing the number of MCP channels. A simple calculation for maximum possible current density with a 1200 channel $/ \mathrm{cm}^{2} \mathrm{MCP}$, placed over a cathode with 1200 uniformly functioning CNTs, would be $\sim 1.46 \mathrm{kA} / \mathrm{cm}^{2}$, neglecting space charge limitations. Clearly this level of emission is far greater than what is needed for the electron lens application, but it does offer a highly comforting margin to account for sub-standard emitters and/or to allow the lesser challenge of building a cathode with fewer channels $/ \mathrm{cm}^{2}$. A satisfactory goal for the electron lens application would be a controllable emission of 2-4 mA per channel in an ensemble of 800-1200 uniformly-functioning channels $/ \mathrm{cm}^{2}$, and a cathode with overall area of about $1 \mathrm{~cm}^{2}$.

This work was sponsored by the US Department of Energy, Office of High Energy Physics, under STTR Grant \# DE-FG02-08ER 86354, 
Omega-P, Inc.

Final Report on STTR Phase I grant DE-FG02-08ER 86354

Topic 49b: Advanced concepts and technology for high energy accelerators:

NOVEL DEVICE AND INSTRUMENTATION DEVELOPMENT

\section{REFERENCES}

1. V.Shiltsev et al., Phys. Rev. STAB 2, 071001 (1999).

2. V.Shiltsev et al., Phys. Rev. STAB 8, 101001 (2005).

3. V.Shiltsev et al. PAC2007, p.728.

4. W. Zhu, C. Bower, O. Zhou, G. Kochanski, S. Jin, "Large current density from carbon nanotube field emitters," Appl. Phys. Lett., 1999, v. 75, pp. 873-875 / K.A. Dean and B.R. Chalamala, "Current saturation mechanisms in carbon nanotube field emitters," Appl. Phys. Lett., 2000, v. 76, pp. 375-377.

5. W.B. Choi, D. S. Chung, J.H. Kang, H.Y. Kim, Y.W. Jin, I.T. Han, Y.H. Lee, J.E. Jung, N.S. Lee, G. S. Park, and J.M. Kim, "Fully sealed, high-brightness carbon nanotube field emission display", Appl. Phys. Lett. 753129 Nov. (1999).

6. K. Teo, L. Hudanski, J Schnell, G. Amaratunga, W. Milne, "Carbon nanotubes as cold cathodes", Nature 2005, 437968.

7. V.V. Zhirnov, C.L. Rinne, G. Wojak, J.J. Hren, "Analysis of high-current yield of diamond-based field emitters," Materials Research Society Symposium - Proceedings, 2000, v. 558, pp. 583-588.

8. Seelaboyina. R., Lahiri, I and Choi, W. B. (2009), Nanoletters (Submitted).

9. Seelaboyina. R., Lahiri, I and Choi, W. B. (2009), (In preparation).

10. Fan S S, Chapline M G, Franklin N R, Tombler T W, Cassell A M and Dai H, Self-Oriented Regular Arrays of Carbon Nanotubes and Their Field Emission Properties, Science(1999) 283 512.

11. Seelaboyina, R., Bodepalli, S., Park, J., Noh, K., Jeon, M., and Choi, W. B., Enhanced field emission from aligned multistage carbon nanotube emitter arrays, Nanotechnology (2008), 19 065605 .

12. Bruining, H.; Githens, Sherwood, Physics and Applications of Secondary Electron Emission (1954), Pergamon Press.

13. Seelaboyina, R., Huang, J. and Choi, W.B., Enhanced field emission of thin multiwall carbon nanotubes by electron multiplication from microchannel plate, Appl. Phys. Lett.(2006), 88 194104 and Virtual Journal of Nanoscale Science \& Technology (2006), 1321.

14. Seelaboyina, R., and Choi, W. B., Recent Progress of Carbon Nanotube Field Emitters and Their Application, Recent Patents in nanotechnology (2007), 1238

15. Barker. R. J., Booske. J. H., Luhmann, N. C., and Nusinovich. G. S., Modern Microwave and Millimeter-Wave Power Electronics (2005),IEEE Press.

16. J.K. Ha, B.H. Chung, S.Y. Yan, J.O. Choi, Drastic changes in the field emission characteristics of a Mo-tip field emitter array having PH3-doped a-Si:H as a resistive layer material throughout vacuum packaging processes in a field emission display, J. Vac. Sci. Technol. B, 2002, v. 20, pp. 2080-2084. 
Omega-P, Inc.

Final Report on STTR Phase I grant DE-FG02-08ER 86354

Topic 49b: Advanced concepts and technology for high energy accelerators:

NOVEL DEVICE AND INSTRUMENTATION DEVELOPMENT

17. J.K. Ha, B.H. Chung, S.Y. Han, J.O. Choi, H.G. Kim, Novel resistive layer structure using via holes of an insulating interdielectric as a current path, J. Vac. Sci. Technol. B, 2001, v. 19, pp. 929-932.

18. Y.S. Choi, J.H. Kang, Y.J. Park, W.B. Choi, C.J. Lee, S.H. Jo, C.G. Lee, J.H. You, J.E. Jung, N.S. Lee, J.M. Kim, An under-gate triode structure field emission display with carbon nanotube emitters, Diamond and Related Materials, 2001, v. 10, pp. 1705-1708.

19. I.H. Kim, J.D. Lee, C.W. Oh, J.W. Park, and B.G. Park, Metal-oxide-semiconductor field effect transistor-controlled field emission display, J. Vac. Sci. Technol. B, 2003, v. 21, pp. 519522.

20. M. Nagao, D. Nicolaescu, T. Matsukawa, S. Kanemaru, J. Itoh, T. Sato, Y. Sato, N. Wada, Metal-oxide-semiconductor field-effect transistor-structured Si field emitter array with a built-in ring gate lens, J. Vac. Sci. Technol. B, 2003, v. 21, pp. 495-499.

21. Pavlov, G. V.,Effect of the Space Charge of Emitted Electrons on Field Electron Emission , Technical Physics (2004), 49 72-79.

22. Feng, Y., Verboncoeur, J. P. and Lin, M. C., Solution for space charge limited field emission current densities with injection velocity and geometric effects corrections, Physics of Plasmas (2008), 15043301.

23. Minoux. E, and group et al., Achieving High-Current Carbon Nanotube Emitters, Nanoletters (2005), 5 2135-2138.

24. J. E. Graebner, S. Jin, G. W. Kammlott, J. A. Herb, and C. F. Gardinier, Large anisotropic thermal conductivity in synthetic diamond films, Nature, 1992, v. 359, p. 401.

25. X. E. Lin, Advanced Accelerator Concepts: Eighth Workshop, edited by Wes Lawson, AIP Conf. Proc. No. 472 (AIP, New York,1999), pp. 676-685. 